\title{
A COMMUTATIVITY THEOREM FOR RINGS
}

\author{
M. CHACRON ${ }^{1}$
}

\begin{abstract}
Let $R$ be any associative ring. Suppose that for every pair $\left(a_{1}, a_{2}\right) \in R \times R$ there exists a pair $\left(p_{1}, p_{2}\right)$ such that the elements $a_{i}$ $-a_{i}^{2} p_{i}\left(a_{i}\right)$ commute, where the $p_{i}^{\prime}$ 's are polynomials over the integers with one (central) indeterminate. It is shown here that the nilpotent elements of $\boldsymbol{R}$ form a commutative ideal $N$, and that the factor ring $R / N$ is commutative. This result is obtained by the use of the concept of cohypercenter of a ring $R$, which concept parallels the hypercenter of a ring.
\end{abstract}

Introduction. Let $R$ be any associative ring with center $Z$. Let $p(t)$ be a polynomial over the integers in one indeterminate. In the early 1950's, I. N. Herstein proved that if $R$ is subject to the condition $x-x^{2} p_{x}(x) \in Z$, all $x \in R$, then $R=Z$ [3]. More recently, Herstein has shown a theorem solving a longstanding question, which asserts that if $R$ is subject to the condition $x_{1}^{n_{1}} \cdot x_{2}^{n_{2}}=x_{2}^{n_{2}} \cdot x_{1}^{n_{1}}$, all $x_{i} \in R\left(n_{i} \geqslant 1\right.$ depending on the $x_{i}^{\prime}$ 's), then the ideal commutator of $R$ is nil [5]. This theorem applies to the rings $R$, which are radical over a commutative subring $A$, in the sense that $x^{n(x)} \in A$, all $x \in R$.

One could look at analogous situations with respect to the $x-x^{2} p(x)$ theorem cited above. For instance if the ring $R$ is subject to the condition

$$
x-x^{2} p(x) \in A
$$

(where $A$ is a commutative subring) does it follow, again, that the ideal commutator is, at least, nil? In this paper we prove the following commutativity theorem. Suppose that for each pair $x_{1}, x_{2}$ there exists a pair of polynomials $p_{1}(t), p_{2}(t)$ such that the elements $x_{i}-x_{i}^{2} p_{i}\left(x_{i}\right)$ commute $(\mathrm{C})$. Then the ideal commutator of $R$ is nil, and the nilpotent elements form a commutative ideal. Thus $R$ satisfies a multilinear identity of degree 4 (Theorem 3 ). This result applies obviously to the rings subject to condition $\left(\mathrm{C}_{0}\right)$ (since $\left(\mathrm{C}_{0}\right)$ is a stronger condition).

Conventions. All polynomials are polynomials over the integers $\mathbf{Z}$ with the indeterminate $t$. We denote by $\delta$ the set of polynomials $g(t)$ of the form $g=t-t^{2} p(t)$. Clearly $\mathcal{E}$ is a multiplicatively closed subset (under composition). Given the ring $R$, we denote by $Z$ the center of $R$, by $J$ the Jacobson

Received by the editors December 2, 1975

AMS (MOS) subject classifications (1970). Primary 16A70.

Key words and phrases. Commutator, polynomials, quasi-regular elements, subgroups preserved re quasi-inner automorphisms.

1 This Research was supported by NRC of Canada grant A7876. 
radical of $R$, by $N$ the prime radical of $R$. If $X$ is a subset of $R, C_{R}(X)$ $=\{a \in R, a x=x a$, all $x \in X\}$. The commutator $x_{1} x_{2}-x_{2} x_{1}$ of the pair $\left(x_{1}, x_{2}\right)$ is denoted by $\left[x_{1}, x_{2}\right]$. Finally the rings considered here need not be with 1 .

1. Preliminary results. We collect some of the facts to be used for our main result (Theorem 3). Remarks $1-4$ are known and easy. Although Remark 8 is essentially proven in [4], we have given its complete proof for convenience of the reader.

REMARK 1 (JACOBI'S IDENTITY). $[x y, a]=[x, a] y+x[y, a]$. If then $[x, a]=0$ it follows that $[x y, a]=x[y, a]$.

REMARK 2. If $I$ is a right ideal of $R, I \cdot\left[C_{R}(I), R\right]=0$.

REMARK 3. If $R$ is prime, and if $I \neq 0, C_{R}(I)=Z$.

REMARK 4. If $R$ is prime, and if $I$ is commutative, $R=Z$.

Recall that an element $x_{0}$ of $R$ such that $x_{0}+x_{0}^{\prime}+x_{0} x_{0}^{\prime}=0$ for some $x_{0}^{\prime}$ commuting with $x_{0}$ is called quasi-regular. Then $1+x_{0}$ is invertible with inverse $1+x_{0}^{\prime}$ (formally). The quasi-inner automorphism $x \mapsto x+x_{0} x+x x_{0}^{\prime}$ $+x_{0} x x_{0}^{\prime}$ is denoted as usual by $x \mapsto\left(1+x_{0}\right) x\left(1+x_{0}\right)^{-1}$.

REMARK 5 [4]. If $x_{0}$ is quasi-regular,

$$
\left[x_{0}, a\right]\left(1+x_{0}\right)^{-1}=a-\left(1+x_{0}\right) a\left(1+x_{0}\right)^{-1} .
$$

REMARK 6 [4]. If $A$ is an additive subgroup of the ring $R$, which is preserved re quasi-inner automorphisms, and if $I$ is a quasi-regular right ideal, then $A \cap I$ $=0$ implies $A \subseteq C_{R}(I)$.

REMARK 7 [4]. If $A$ is as in Remark 6, and if $A$ contains no nilpotent elements, for any $a \in A$ with $a u=0$, we have $u R a^{2}=0$. Thus if $R$ is prime, $A$ has no divisors of zero on $R$.

Remark 8 (Brauer, Herstein). Suppose that the sequence $\left(x, z, b_{1}, b_{2}\right)$ in $R$ has the following properties:

(i) $z, b_{1}, b_{2}$ commute pairwise;

(ii) $x,-z \neq 0, z x$ are quasi-regular;

(iii) If $c_{i}=\left[x, b_{i}\right]$ is a zero divisor then $c_{i}=0$;

(iv) $\left[(1+x) b_{2}(1+x)^{-1}, b_{1}\right]=0,\left[(1+z x) b_{2}(1+z x)^{-1}, b_{1}\right]=0$.

Then at least one of the $c_{i}$ 's must be 0 .

Proof. Let $a_{1}=(1+x) b_{2}(1+x)^{-1}$ and $a_{2}=(1+z x) b_{2}(1+z x)^{-1}$. We have by (iv),

$$
\text { (v) }\left[a_{1}, b_{1}\right]=\left[a_{2}, b_{1}\right]=0
$$

and we have

$$
(1+x) b_{2}=a_{1}(1+x), \quad(1+z x) b_{2}=a_{2}(1+x) .
$$

An elementary operation gives

$$
(z-1) b_{2}=z a_{1}-a_{2}+\left(z a_{1}-a_{2} z\right) x .
$$

Now by (i) both $b_{2}$ and $z$ commute with $b_{1}$, so, $\left[(z-1) b_{2}, b_{1}\right]=0$. Also $z$, 
$a_{1}, a_{2}$ commute all with $b_{1}((\mathrm{v}))$, thus $\left[z a_{1}-a_{2}, b_{1}\right]=0$. Since by Jacobi's identity $\left[\left(z a_{1}-a_{2} z\right) x, b_{1}\right]=\left(z a_{1}-a_{2} z\right)\left[x, b_{1}\right]$, we see that $\left(\mathrm{E}_{2}\right)$ implies

$$
\left(z a_{1}-a_{2} z\right) \cdot\left[x, b_{1}\right]=\left(z a_{1}-a_{2} z\right) c_{1}=0 .
$$

If then $c_{1} \neq 0$ is a nonzero divisor, $\left(\mathrm{E}_{3}\right)$ gives $z a_{1}-a_{2} z=0$. Going back to $\left(E_{2}\right)$ we get

$$
b_{2}(z-1)=(z-1) b_{2}=z a_{1}-a_{2}=a_{2} z-a_{2}=a_{2}(z-1),
$$

that is, $\left(b_{2}-a_{2}\right)(z-1)=0$, so, $(z-1)^{-1}\left(b_{2}-a_{2}\right)(z-1)=0$, consequently $b_{2}=a_{2}$. From the second equation in $\left(\mathrm{E}_{1}\right)$ follows $\left[b_{2}, z x\right]=0$. Since $\left[b_{2}, z\right]=0$ we get $z\left[b_{2}, x\right]=0$, so, $z \cdot c_{2}=0$. Since $c_{2}$ is a nonzero divisor or 0 and since $z \neq 0, c_{2}=0$ necessarily, thereby proving Remark 8 .

REMARK 9. Let $R$ be a prime ring having a radical $(J \neq 0)$. Any commutative subgroup $A$ of $R$ containing no nilpotent elements, which is preserved re quasiinner automorphisms, must be contained in the center.

Proof. If $A \cap J=0$, then by Remark 6, $A \subseteq C_{R}(J)$, and by Remark 3, $C_{R}(J)=Z$, so $A \subseteq Z$. If, on the other hand, $A \cap J \neq 0$, choose $z \neq 0$ in $A \cap J$, and any $a \in A, x \in J$. If $b_{1}=b_{2}=a$, the sequence $\left(x, z, b_{1}, b_{2}\right)$ has all the requirements as in Remark 8. Let us show, for example (iii). Let $c=[x, a]$. If $c \neq 0$, then $c(1+x)^{-1} \neq 0$ is in $A$ (Remark 5). By Remark 7, $A$ has no divisors on $R$, thus $c(1+x)^{-1}$ is a nonzero divisor. It follows that $c$ is a nonzero divisor (or zero). Applying Remark 8 we see that $c=0$ necessarily, that is, $[x, a]=0$. Thus $A \subseteq C_{R}(J)=Z$ as wished.

REMARK 10. Let $\mathcal{E}_{0}$ be a closed set of polynomials. Suppose that for every $\left(a_{1}, a_{2}\right)$ there is $\left(g_{1}, g_{2}\right) \in \varepsilon_{0} \times \varepsilon_{0}$ such that $\left[g_{1}\left(a_{1}\right), g_{2}\left(a_{2}\right)\right]=0$. Let $a, x \in R$ with the following conditions.

(i) $-a, x$, ax are quasi-regular;

(ii) $\left[x, \mathcal{E}_{0}(a)\right]$ has no proper divisors of zero on $R$.

Then for some $g \in \mathcal{E}_{0},[x, g(a)]=0$.

Proof. Applying $\left(\mathrm{C}_{0}\right)$ for $\left((1+x) a(1+x)^{-1}, a\right)$ we get for some $g_{1}, h_{1}$ $\in \mathcal{E}_{0}$,

$$
\left[(1+x) g_{1}(a)(1+x)^{-1}, h_{1}(a)\right]=0 .
$$

Applying $\left(\mathrm{C}_{0}\right)$ for $\left[(1+a x) g_{1}(a)(1+a x)^{-1}, h_{1}(a)\right]$ we get for some $g_{2}, h_{2}$ $\in \mathcal{E}_{0}$,

$$
\left[(1+a x) g_{2}\left(g_{1}(a)\right)(1+a x)^{-1}, h_{2}\left(h_{1}(a)\right)\right]=0 .
$$

Setting $b_{1}=h_{2}\left(h_{1}(a)\right), b_{2}=g_{2}\left(g_{1}(a)\right), z=a$, we see that $\left(x, z, b_{1}, b_{2}\right)$ satisfies the hypothesis in Remark 8 , so $\left[x, b_{1}\right]=0$ or $\left[x, b_{2}\right]=0$, with $b_{i}=g(a)$, some $g \in \varepsilon_{0}$.

2. Cohypercenter. The analog with respect to condition $(C)$ (see Introduction) of Herstein's hypercenter [4] is the following set $T=T(R)$. 
Definition. $a \in T$ if and only if given $x \in R$ there exists $p(t)$ such that $\left[a, x-x^{2} p(x)\right]=0$, where $p(t)$ is a polynomial having integral coefficients depending on $(a, x)$.

Let us call $T$ the cohypercenter of $R$. We wish to get the property $T=Z$ for, at least, the class of semiprime rings.

REMARK 11. Given $a, b \in T$ and $x \in R$, there is $p(t)$ such that $\left[a, x-x^{2} p(x)\right]$ $=\left[b, x-x^{2} p(x)\right]=0$.

Proof. Since $a \in T$ there is $g_{1} \in \mathcal{E}\left(=\left\{t-t^{2} \mathbf{Z}[t]\right\}\right)$ such that $\left[a, g_{1}(x)\right]$ $=0$. Since $b \in T$ there is $g_{2} \in \mathcal{E}$ such that $\left[b, g_{2}\left(g_{1}(x)\right)\right]=0$. If $g=g_{2} \circ g_{1}$, then $g \in \mathcal{E}$, and $[a, g(x)]=[b, g(x)]=0$.

REMARK 12. $T$ is a commutative subring evidently preserved re quasi-inner automorphisms.

Proof. Given $a, b \in T$ and $x$ there is $g \in \mathcal{E}$ such that $[a, g(x)]=[b, g(x)]$ $=0$ (Remark 11). Thus $[a \pm b, g(x)]=[a b, g(x)]=0$, and $x \in C_{R}\left(T_{0}\right)$, where $T_{0}$ is the subring of $R$ generated by $a$, and $b$ shows that $T$ is a subring and that $x-x^{2} p_{x}(x)=g_{x}(x) \in Z\left(T_{0}\right)$, all $x \in T_{0} \subseteq R$. By Herstein [3], $T_{0}$ is commutative. Therefore $[a, b]=0$, all $a, b \in T$, that is, $T$ is commutative.

Remark 13 [2, Lemma 2]. Let $V$ be a space over the division ring $D$. If $f$ is a linear transformation sending each $\vec{v} \in V$ to $\vec{v} \lambda, \lambda \in D$, depending on $\vec{v}$, and if $\operatorname{dim} V>1$, then $f$ is induced by a scalar (central element $\lambda_{0}$ of $D$ ).

THEOREM 1. If $R$ is any semiprime ring, the cohypercenter of $R$ is precisely the center of $R$.

Proof. We prove the assertion by a step-by-step reduction from division rings to the considered rings.

First if $R$ is a division ring, it is clear that $T=Z$ (Remark 9, and Brauer, Cartan, Hua result).

Next if $R$ is a primitive ring, which is not a division ring, then by the density theorem, $R$ acts densely on a space $V$ over the division ring $D$ with a dimension $>1$. Let $\vec{v} \in V$ and let $a \in T$. If $\vec{v}$ and $\vec{v} a$ were not collinear, by the density action of $R$, there is $x \in R$ with $\vec{v} x=0$ and $\vec{v}$ ax $=\vec{v}$. Since $a \in T$, there is $y=g(x)=x-x^{2} p(x)$ with $[a, y]=0$, whence, $\vec{v}$ ay $=\vec{v} y a$. Now

$$
\vec{v} \text { ay }=\vec{v} \text { ax }-\vec{v} \text { ax }(x p(x))=\vec{v}-\vec{v}(x p(x))=\vec{v} ;
$$

but, $\vec{v} y a=\vec{v} x(1-x(p(x))) a=\overrightarrow{0}$, a contradiction. This shows that $\vec{v} a$ $=\vec{v} \lambda$, some $\lambda \in D$. By Remark 13, $a$ is induced by a scalar in $D$. Therefore $a \in Z(R)$.

Next if $R$ is semisimple $(J=0)$, then $R$ is a subdirect product of primitive rings $\bar{R}$. By the above $\bar{T}=T(\bar{R})=\bar{Z}=Z(\bar{R})$. Since $T$ maps into $\bar{T}, T$ is central in $\bar{R}$. Therefore $T=Z$.

Next suppose that $R$ is prime but not semisimple. If $a^{2}=0$ with $a \in T$, we claim that $a=0$. In fact if $a \neq 0, I=a R \neq 0$. Since $a \in T$, for every $x \in R$, there is $g \in \mathcal{E}$ such that $[a, g(a x)]=0$. Thus for some $p(t)$, axa $=(a x)^{2} p(a x) a$. Multiplication on the right by $x$ gives $(a x)^{2}=(a x)^{3} p(a x)$. 
Therefore $y^{2}=y^{3} p_{y}(y)$, all $y \in I$. Now if $I$ were nil, $y^{2}=0$, all $y \in I$, so, $R$ would contain a nilpotent ideal (Levitski's result), a contradiction. This shows that the $\pi$-regular ring $I$ must contain some idempotent $e \neq 0$. If $R_{0}=e R e$, this is a nonzero prime ring verifying again $y^{2}=y^{3} p_{y}(y)$ (since $R_{0} \subseteq e R$ $\subseteq I$ ). Since by [2], $R_{0}$ satisfies a polynomial identity and since $R_{0}$ is $\pi$-regular, $R_{0}$ is the ring of matrices over a division ring. Thus $R_{0}$, whence $R$, have nonzero socles, contrary to the choice of $R$. This shows that $a=0$ necessarily, and $T$ contains no nilpotents, By Remarks 12 and $9, T=Z$ follows.

All in all, we have shown that if $R$ is prime, $T=Z$. We go back to the semiprime ring $R$. Since $R$ is a subdirect product of prime rings satisfying the desired conclusion, it follows that $T=Z$, thereby proving the theorem.

\section{The main result.}

REMARK 14. If $R$ satisfies (C), any nilpotent element a must belong to $T$.

Proof. Given $a, b \in R$, we claim that for any given integer $n_{0}$ there is $n \geqslant n_{0}$ such that $\left[a-a^{n} p_{1}(a), b-b^{2} p_{2}(b)\right]=0$. In fact, by the basic property, $\left[a-a^{2} p_{1}(a), b-b^{2} p_{2}(b)\right]=0$. If $a_{1}=a^{2} p_{1}(a), b_{1}=b-b^{2} p_{2}(b)$, there are $p_{11}, p_{22}$ such that

$$
\left[a_{1}-a_{1}^{2} p_{11}(a), b_{1}-b_{1}^{2} p_{22}\left(b_{1}\right)\right]=0 .
$$

Now the first commutator relation gives $\left[a-a_{1}, b_{1}-b_{1}^{2} p_{22}\left(b_{1}\right)\right]=0$. Adding to the preceding, we get for some $p_{21}(t),\left[a-a^{4} \cdot p_{21}(a), b-b^{2} p_{22}(b)\right]=0$. Continuing in this way, we see that for any $n_{0}, n=2^{n_{0}}$ will do. If then $a$ is nilpotent of index $n_{0}$, for any $b \in R$,

$$
\left[a-a^{2^{n_{0}}} p_{n_{0}}^{\prime}(a), b-b^{2} p_{2}^{\prime}(b)\right]=\left[a, b-b^{2} p_{2}^{\prime}(b)\right]=0
$$

tell us that $a \in T$.

THEOREM 2. If $R$ is a semiprime ring satisfying (C), then $R$ is commutative.

Proof. First suppose that $R$ is a division ring. Let $a, x \in R$. If condition (i) in Remark 10 does not hold, then evidently $[a, x]=0$. If, on the other hand, (i) holds, by Remark $10,[x, g(a)]=0$, some $g \in \mathcal{E}$. This shows that for any $x \in R, x \in T$. By Theorem $1, R=Z$.

Next suppose that $R$ is primitive. Every homomorphic image $\bar{R}$ of a subring of $R$ inherits condition (C), which in view of Remarks 14 and 12, tells us that in $\bar{R}$ the nilpotent elements commute. Since this is patently false for the matrix rings over division rings of rank $>1$, by a routine argument, $R$ must be a division ring, so, by the above, $R$ must be a field.

Having proved the assertion for the primitive rings we derive, as before, that it holds for the semisimple rings.

Next suppose that $R$ is prime but not semisimple. Since $R$ is evidently semiprime, $T=Z$ (Theorem 1). By Remark 14, $R$ has no nilpotent elements. It follows that $R$ has no divisors of zero. Thus the $\operatorname{ring} J$ is a ring subject to condition (C), and has no divisors of zero. Let $x, a \in J$. The pair $(a, x)$ satisfies 
the hypothesis of Remark 10. Consequently for some $g \in \mathcal{E},[x, g(a)]=0$. This shows that $x \in T(J)$, all $x \in J$. By Theorem $1, J=Z(J)$ follows, and by Remark $4, R=Z$.

Having proved the assertion for the prime rings we derive the desired result. Let $R$ be any ring subject to condition (C). Since the factor ring $R / N$ inherits (C) and is semiprime, Theorem 2 applies and yields $R / N$ commutative, whence, $R / N$ has no nilpotent elements. This means that $N$ is the set of nilpotent elements of $R$. By Remark 14, $\subseteq \subseteq T$ is also commutative. If then $I$ is the ideal commutator of $R, I \subseteq N$ is commutative, so $R$ satisfies the identity $\left[\left[x_{1}, x_{2}\right],\left[x_{3}, x_{4}\right]\right]=0$. Summarizing we get the main result.

THEOREM 3. Suppose that for each pair $x_{1}, x_{2}$ in the ring $R$ there exists a pair $\left(p_{1}(t), p_{2}(t)\right)$ of polynomials with integral coefficients such that the elements $x_{i}-x_{i}^{2} p_{i}\left(x_{i}\right)$ commute. Then the ideal commutator of $R$ is nil, and the nilpotent elements form a commutative ideal.

One final remark is in order.

REMARK 15 (A noncommutative ring as in Theorem 3 ). We observed earlier that the rings $R$ subject to the condition $\left(\mathrm{C}_{0}\right)$ (see Introduction) satisfy $(\mathrm{C})$, so, the conclusion in Theorem 3 holds for such a class. One could wonder if the latter rings are commutative. If $R$ is the ring of matrices $R=\left(\begin{array}{c}F F \\ 0\end{array}\right)$ over the field $F$, and if $F$ is algebraic over a finite field, it was verified in [1] that for every $x \in R$, either $x^{2}=0$ or $x=x^{n(x)+1}, n(x) \geqslant 1$. Thus $R$ satisfies condition $\left(\mathrm{C}_{0}\right)$ re the commutative subring, even commutative ideal, $A=J=\left(\begin{array}{ll}0 & F \\ 0 & 0\end{array}\right)$. Here $N=J$ is the set of nilpotent elements, and $R / N \approx F \times F$ is in fact commutative. Is this example the most general one for the noncommutative subdirectly irreducible rings $R$ subject to (C)? If true this would give that the ideal commutator of any ring $R$ with $(\mathrm{C})$ is a square-zero ideal.

\section{REFERENCES}

1. M. Chacron and G. Thierrin, An algebraic dependence over the quasi-centre, Ann. Math. Pura Appl. (to appear).

2. M. Chacron, On a theorem of Herstein, Canad. J. Math. 21 (1969), 1348-1353. MR 41 \#6905.

3. I. N. Herstein, The structure of a certain class of rings, Amer. J. Math. 75 (1953), 864-871. MR 15, 392.

4. - On the hypercenter of a ring, J. Algebra (to appear).

5. _ A commutativity theorem, J. Algebra 38 (1976), 112-118.

Department of Mathematics, Carleton University, Ottawa, Ontario, Canada 